\title{
Tandem bispecific broadly neutralizing antibody - a novel approach to HIV-1 treatment
}

\author{
Guido Ferrari \\ Department of Surgery, Duke University Medical Center, Durham, North Carolina, USA.
}

\begin{abstract}
The last decade has led to a significant advance in our knowledge of HIV-1 latency and immunity. However, we are still not close to finding a cure for HIV-1. Although combination antiretroviral therapy (cART) has led to increased survival, almost close to that of the general population, it is still not curative. In the current issue of the $J C I$, Wu et al. studied the prophylactic and therapeutic potential of an engineered tandem bispecific broadly neutralizing antibody (bs-bnAb), BiIA-SG. This bnAb's breadth and potency were highly effective in protection and treatment settings, as measured by complete viremia control following direct infusion, as well as elimination of infected cells and delay in viral rebound when delivered with a recombinant vector. These observations underscore the need for the clinical development of BilA-SC for the prevention of HIV-1.
\end{abstract}

ated structure of the HIV-1 envelope present on the viral membrane, referred to as the closed trimeric structure of the gp120/gp41 complex. This closed state is the most infectious among the different conformational states of the envelope present on HIV-1 virions $(6,7)$. Similar structures can also be exposed on the membrane of the infected cells at the time of virus budding (8).

\section{Broadly neutralizing Abs offer hope}

Over the years, several studies have exploited the ability of bnAbs to confer protection from infection, to control virus replication, and to reduce the size of the latently infected cells' reservoir (review in ref. 9). The original passive protection studies in animal models indicated that monoclonal $\mathrm{Ab}(\mathrm{mAb})$ combinations are usually more effective in preventing simian human immunodeficiency virus (SHIV) infection in nonhuman primates (10-13). More recently, bnAb combinations have also been tested for their potential activity in reducing viremia, viral rebound in animal models, and in exploratory clinical trials. In the humanized mouse model, a combination of the CD4 binding site 3BNC117 (14), V3 glycan 10-1074 (15), and V1V2 glycan PG16 (16) bnAbs induced decreased viremia in approximately $50 \%$ of the mice, and substantially delayed virus rebound compared with animals treated with cART (17). Moreover, a decline in the level of cellular-associated DNA occurred only in the aviremic mice treated with the 3-mAb cocktail. Therefore, bnAbs can significantly impact both plasma viremia and the pool of latently infected cells through recognition of HIV-1 Env on the host cell membrane (17). Similar observations have been reported in the nonhuman primate model in which bnAbs successfully reduced the level of plasma viremia during chronic (18-20) and acute (21) SHIV infection. Additionally, bnAb combinations have been shown to reduce the size of the pool of latently infected cells $(19,21)$. 
A BilA-SG

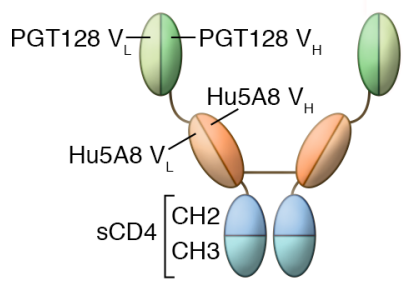

B Intraspike cross-linking

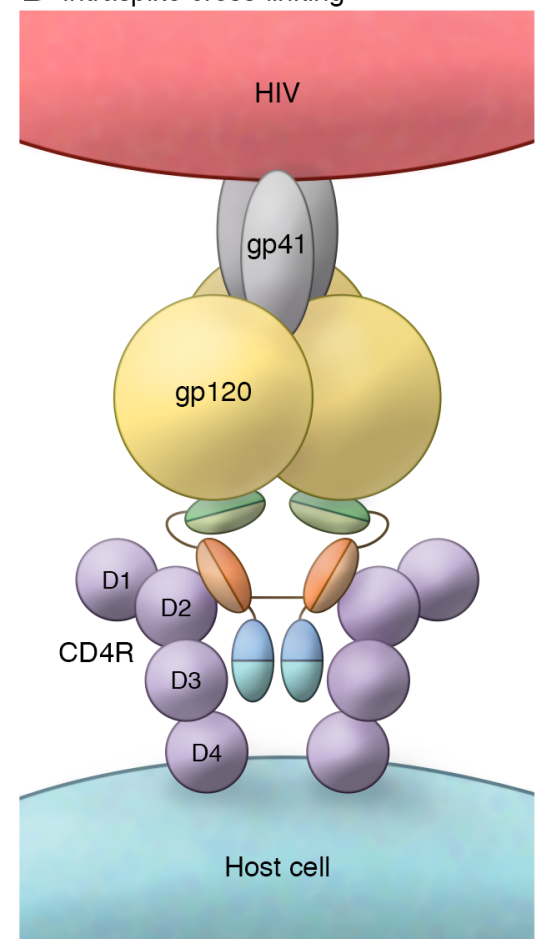

C Interspike cross-linking

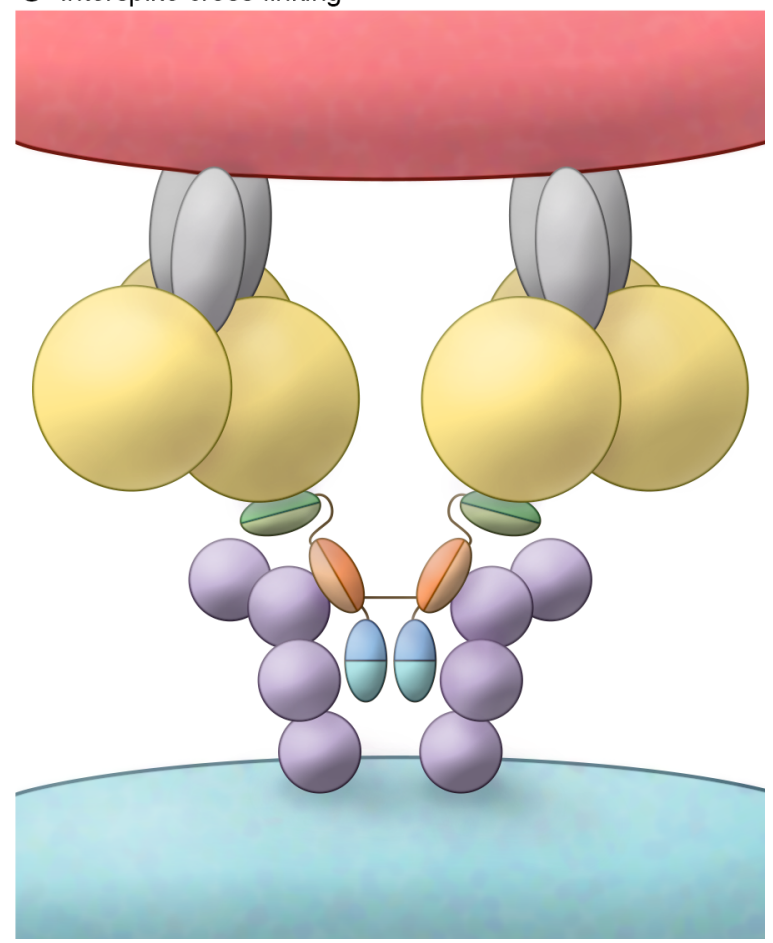

Figure 1. Schematic diagram showing binding of the BilA-SG antibody. (A) Engineered tandem bispecific bnAb BilA-SG antibody with scFv domain(s) for binding to HIV-1 gp160 trimer (green VH/VL chain of PCT128) and to soluble CD4 (orange VH/VL chain of Hu5A8) via 20-mer linkers. Two possible interactions are predicted for the molecule: intraspike cross-linking (B) and interspike cross-linking (C). scFv, single-chain variable fragment; $\mathrm{VH}$, heavy-chain variable region; VL, light-chain variable region.

The efficacy of individual bnAb infusions was recently tested in human trials and appeared to have a beneficial effect on the control of plasma viremia (22). However, in 2 different regimens, resistant virus isolates were either observed before treatment and hampered the therapeutic effect of the bnAb (23), or appeared following infusion of the bnAbs during analytical treatment interruption (24). Taken together, these studies have thus far indicated that development of mAb-based molecules with several epitope specificities is a desirable goal to circumvent HIV-1 envelope sequence diversity and propensity to escape.

\section{A novel approach toward the broadly neutralizing Abs}

$\mathrm{Wu}$ and collaborators (25) decided to take a different approach to circumvent the diversity of HIV-1 envelopes. They based their molecule on 1 single gene-encoded tandem bs-bnAb, namely BiIA-SG, where 2 single-chain variable fragment ( $\mathrm{scFv}$ ) binding domains against the V3 glycan epitope recognized by the PGT128 mAb and the CD4 receptor recognized by the Hu5A8 mAb are simultaneously present (Figure 1). This represents a radically different approach compared with a predecessor of this molecule reported by Huang and collaborators (26) that included a single scFv for each anti-HIV-1 envelope broadly neutralizing monoclonal antibody (bnmAb) against the CD4 receptor. The aspects related to the new class of molecules discussed herein do not include obvious considerations on the pharmacokinetic, tissue distribution, and ability to induce anti-drug antibody responses, since these will have to be addressed in further dedicated studies.

As reported by Wu et al. (25), the neutralizing breadth and potency of the new BiIA-SG molecule are definitely higher than those of the original neutralizing PGT128 mAb, or the BiIA-DG with a single scFv domain. The authors suggest that the improved activity of the BiIA-SG may be due to the ability of the anti-CD4 arm to bring the PGT121 arm in proximity to the HIV-1 Env. In addition, this effect may also be due to the presence of 2 binding sites that favor the binding of the molecule to the targeted antigens. In developing a new class of therapeutics, this aspect could be relevant to improve the performance of bispecific molecules such as those based on the potent class of membrane proximal epitope region-specific (MPER-specific) mAbs that do need a simultaneous bivalent binding to the membrane lipid layer and to the HIV-1 envelope (27). It is interesting that a similar molecule was originally designed and characterized to include the MPER 10e8 specificity and was also developed into a trispecific molecule (28). Ultimately, it will be interesting to observe if this newly engineered molecule can address the need for the simultaneous recognition of 2 different regions of the HIV-1 envelope to broaden the breadth and prevent escape. These new molecules could represent the benchmark for comparison with the recently reported trispecific mAb-based molecules that are also aimed at increasing breadth and potency of antiHIV-1 activity (29).

The authors also demonstrate that the BiIA-SG molecule is able to protect from 
infection and control virus rebound in the humanized mouse model for HIV-1 infection. Of note, the molecule, delivered as an adeno-associated virus construct, led to a reduction of $\mathrm{P} 24^{+} \mathrm{T}$ cells in the blood and spleen.

\section{Concluding remarks}

Additional information about the activity of BiIA-SG against the so-called sanctuary for HIV-1 in the latent reservoir will have to be obtained before designing studies to prevent the appearance of escape mutants and to reduce the virus reservoir in tissues such as lymph nodes, the GI tract, and the central nervous system. If the mechanism of action of the BiIA-SG is related to its broadly neutralizing function, it still remains to be determined how this molecule will perform against the circulating HIV-1 isolates, taking into account HIV-1 diversity and the dichotomy already observed between in vitro breadth of individual bnAbs and the presence of escape mutants $(23,24)$.

In addition to the neutralization activity, bnAbs can significantly impact both plasma viremia and the pool of latently infected cells through recognition of the HIV-1 Env on the host cell membrane (17) and engagement of $\mathrm{Fc} \gamma$-R-bearing cells (30). Therefore, the possibility of further enhancing the activity of this promising class of immunotherapeutics needs to be explored by designing bnAbs that can engage $\mathrm{Fc} \gamma$-R-bearing cells and facilitate the killing of latently infected cells.

\section{Acknowledgments}

This manuscript was supported by the Center For AIDS Research, Immunology Core, Duke University Medical Center (AI064518).

Address correspondence to: Guido Ferrari, Duke University Medical Center, $915 \mathrm{La}$ Salle Street, SORF Building, Room 208, Durham, North Carolina 27710, USA. Phone: 919.684.2862; Email: gflmp@ dm.duke.edu.
1. Gaschen B, et al. Diversity considerations in HIV-1 vaccine selection. Science. 2002;296(5577):2354-2360.

2. Korber B, Gaschen B, Yusim K, Thakallapally R, Kesmir C, Detours V. Evolutionary and immunological implications of contemporary HIV-1 variation. Br Med Bull. 2001;58:19-42.

3. Liao HX, et al. Co-evolution of a broadly neutralizing HIV-1 antibody and founder virus. Nature. 2013;496(7446):469-476

4. Gao F, et al. Cooperation of B cell lineages in induction of HIV-1-broadly neutralizing antibodies. Cell. 2014;158(3):481-491.

5. Moody MA, et al. Strain-specific V3 and CD4 binding site autologous HIV-1 neutralizing antibodies select neutralization-resistant viruses. Cell Host Microbe. 2015;18(3):354-362.

6. Poignard $\mathrm{P}$, et al. Heterogeneity of envelope molecules expressed on primary human immunodeficiency virus type 1 particles as probed by the binding of neutralizing and nonneutralizing antibodies. JVirol. 2003;77(1):353-365.

7. Moore PL, et al. Nature of nonfunctional envelope proteins on the surface of human immunodeficiency virus type 1.J Virol.2006;80(5):2515-2528.

8. Pollara J, Bonsignori M, Moody MA, Pazgier M, Haynes BF, Ferrari G. Epitope specificity of human immunodeficiency virus-1 antibody dependent cellular cytotoxicity [ADCC] responses. Curr HIV Res. 2013;11(5):378-387.

9. Margolis DM, Koup RA, Ferrari G. HIV antibodies for treatment of HIV infection. Immunol Rev. 2017;275(1):313-323.

10. Mascola JR, et al. Potent and synergistic neutralization of human immunodeficiency virus (HIV) type 1 primary isolates by hyperimmune anti-HIV immunoglobulin combined with monoclonal antibodies 2F5 and 2G12. J Virol. 1997;71(10):7198-7206

11. Hessell AJ, et al. Broadly neutralizing monoclonal antibodies 2F5 and 4E10 directed against the human immunodeficiency virus type $1 \mathrm{gp} 41$ membrane-proximal external region protect against mucosal challenge by simian-human immunodeficiency virus SHIVBa-L. J Virol. 2010;84(3):1302-1313.

12. Pegu A, et al. Neutralizing antibodies to HIV-1 envelope protect more effectively in vivo than those to the CD4 receptor. Sci Transl Med. 2014;6(243):243ra88.

13. Sholukh AM, et al. Defense-in-depth by mucosally administered anti-HIV dimeric IgA2 and systemic IgG1 mAbs: complete protection of rhesus monkeys from mucosal SHIV challenge. Vaccine. 2015;33(17):2086-2095.

14. Scheid JF, et al. Sequence and structural convergence of broad and potent HIV antibodies that mimic CD4 binding. Science. 2011;333(6049):1633-1637.

15. Mouquet H, et al. Complex-type N-glycan recognition by potent broadly neutralizing HIV antibodies. Proc Natl Acad Sci U S A. 2012;109(47):E3268-E3277.

16. Walker LM, et al. Broad and potent neutralizing antibodies from an African donor reveal a new HIV-1 vaccine target. Science. 2009;326(5950):285-289.

17. Halper-Stromberg A, et al. Broadly neutralizing antibodies and viral inducers decrease rebound from HIV-1 latent reservoirs in humanized mice. Cell. 2014;158(5):989-999.

18. Shingai M, et al. Antibody-mediated immunotherapy of macaques chronically infected with SHIV suppresses viraemia. Nature. 2013;503(7475):277-280

19. Barouch DH, et al. Therapeutic efficacy of potent neutralizing HIV-1-specific monoclonal antibodies in SHIV-infected rhesus monkeys. Nature. 2013;503(7475):224-228

20. Shingai M, et al. Passive transfer of modest titers of potent and broadly neutralizing anti-HIV monoclonal antibodies block SHIV infection in macaques. JExp Med. 2014;211(10):2061-2074.

21. Bolton DL, et al. Human immunodeficiency virus type 1 monoclonal antibodies suppress acute simian-human immunodeficiency virus viremia and limit seeding of cell-associated viral reservoirs. J Virol. 2016;90(3):1321-1332.

22. Caskey M, et al. Viraemia suppressed in HIV-1infected humans by broadly neutralizing antibody 3BNC117. Nature. 2015;522(7557):487-491.

23. Lynch RM, et al. Virologic effects of broadly neutralizing antibody VRC01 administration during chronic HIV-1 infection. Sci Transl Med. 2015;7(319):319ra206.

24. Scheid JF, et al. HIV-1 antibody 3BNC117 suppresses viral rebound in humans during treatment interruption. Nature. 2016;535(7613):556-560.

25. Wu X, et al. Tandem bispecific neutralizing antibody eliminates HIV-1 infection in humanized mice. JClin Invest. 2018;128(6):2239-2251.

26. Huang Y, et al. Engineered bispecific antibodies with exquisite HIV-1-neutralizing activity. Cell. 2016;165(7):1621-1631.

27. Alam SM, et al. Role of HIV membrane in neutralization by two broadly neutralizing antibodies. Proc Natl Acad Sci U S A. 2009;106(48):20234-20239.

28. Steinhardt JJ, et al. Rational design of a trispecific antibody targeting the HIV-1 Env with elevated anti-viral activity. Nat Commun. 2018;9(1):877.

29. Xu L, et al. Trispecific broadly neutralizing HIV antibodies mediate potent SHIV protection in macaques. Science. 2017;358(6359):85-90.

30. Bournazos S, Klein F, Pietzsch J, Seaman MS, Nussenzweig MC, Ravetch JV. Broadly neutralizing anti-HIV-1 antibodies require Fc effector functions for in vivo activity. Cell. 2014;158(6):1243-1253. 